\title{
IMPLANTAÇÃO DO PROCESSO DE ENFERMAGEM EM UNIDADE DE CUIDADOS INTERMEDIÁRIOS
}

\author{
IMPLEMENTATION OF THE NURSING PROCESS \\ IN AN INTERMEDIATE CARE UNIT
}

\section{IMPLEMENTACIÓN DEL PROCESO DE ENFERMERÍA EN UNA UNIDAD DE CUIDADOS INTERMEDIOS}

\author{
Daislan Carvalho ${ }^{1}$ \\ Flávia Emília Cavalcante Valença Fernandes ${ }^{2}$ \\ Gerlene Grudka Lira ${ }^{3}$ \\ Nêmora Lígia de Sousa Santana ${ }^{4}$ \\ Giselle Karine Muniz de Melo ${ }^{5}$ \\ Rita de Kacia de Sousa ${ }^{6}$ \\ Rachel Mola ${ }^{7}$
}

Como citar este artigo: Carvalho D, Fernandes FECV, Lira GG, Santana NLS, Melo GKM, Sousa RK, et al. Implantação do Processo de Enfermagem em unidade de cuidados intermediários. Rev baiana enferm. 2022;36:e43048.

Objetivo: descrever a implantação do Processo de Enfermagem em uma unidade de cuidados intermediários. Método: pesquisa-ação realizada em hospital público de ensino de dezembro de 2019 a março de 2020, contando com quatro capacitações sobre Processo de Enfermagem e Teorias de Enfermagem; apresentação dos instrumentos do Processo de Enfermagem, implantação e acompanhamento. Alterações sugeridas pelos enfermeiros ocorreram mediante observação participante e diário de campo nas visitas in loco. Resultados: relatadas dificuldades no preenchimento inicial dos instrumentos; inexperiência prática; falta de tempo e sobrecarga de trabalho. Os benefícios mencionados foram relacionados à formatação clara e ordenada dos instrumentos; facilidade na aplicação das etapas e segurança na prática assistencial. Considerações finais: a implantação do Processo de Enfermagem no setor ocorreu após a identificação do conhecimento prévio da equipe de Enfermagem, seguida do levantamento do perfil de pacientes, criação dos instrumentos, realização de educação permanente dos profissionais, aplicação e acompanhamento no uso dos instrumentos.

Descritores: Processo de Enfermagem. Teoria de Enfermagem. Registros de Enfermagem. Terminologia Padronizada em Enfermagem. Educação Continuada.

Objective: to describe the implementation of the Nursing Process in an intermediate care unit. Method: action research conducted in a public teaching hospital from December 2019 to March 2020 with four training on Nursing

Enfermeiro. Pesquisador Independente. Petrolina, Pernambuco, Brasil. https://orcid.org/0000-0002-7562-695।.

Enfermeira. Doutora em Inovação Terapêutica. Professora Adjunta da Universidade de Pernambuco. Petrolina, Pernambuco, Brasil. https://orcid.org/0000-00032840-8561.

3 Enfermeira. Mestre em Ciências da Saúde. Professora Assistente da Universidade de Pernambuco. Petrolina, Pernambuco, Brasil. https://orcid.org/0000-00025175-7738.

4 Enfermeira. Mestre em Ciências da Saúde e Biológicas. Enfermeira Assistencial do Hospital Universitário da Universidade Federal do Vale do Rio São Francisco. Petrolina, Pernambuco, Brasil. https://orcid.org/0000-0002-2080-5645.

Enfermeira. Especialista em Segurança do Paciente para Profissionais da Rede de Urgência e Emergência. Enfermeira Assistencial do Hospital Universitário da Universidade Federal do Vale do Rio São Francisco. Petrolina, Pernambuco, Brasil. https://orcid.org/0000-0002-0643-8658.

Enfermeira. Especialista em Obstetrícia. Enfermeira Assistencial do Hospital Universitário da Universidade Federal do Vale do Rio São Francisco. Petrolina, Pernambuco, Brasil. https://orcid.org/0000-000 I-6805-5I 53.

Enfermeira. Doutora em Enfermagem. Professora Adjunta da Universidade de Pernambuco. Petrolina, Pernambuco, Brasil. rachel.mola@upe.br. https://orcid. org/0000-0002-0180-2721. 
Process and Nursing Theories; presentation of nursing process instruments, implementation and follow-up. Changes suggested by the nurses occurred through participant observation and field diary in in loco visits. Results: difficulties in initial filling of instruments were reported, as well as practical inexperience and lack of time and work overload. The benefits mentioned were related to the clear and orderly formatting of the instruments; ease in the application of steps and safety in care practice. Final considerations: the implementation of the Nursing Process in the sector occurred after the identification of the previous knowledge of the nursing team, followed by the survey of the profile of patients, creation of instruments, execution of permanent education of professionals, application and monitoring in the use of the instruments.

Descriptors: Nursing Process. Nursing Theory. Nursing Records. Standardized Nursing Terminology. Continuing Education.

Objetivo: describir la implementación del Proceso de Enfermería en una unidad de cuidados intermedios. Método: investigación-acción realizada en un hospital docente público de diciembre de 2019 a marzo de 2020 con cuatro capacitaciones sobre Procesos de Enfermería y Teorías de Enfermería; presentación de instrumentos del proceso de enfermería, implementación y seguimiento. Los cambios sugeridos por las enfermeras ocurrieron a través de la observación participante y el diario de campo en las visitas in loco. Resultados: se reportaron dificultades en el llenado inicial de los instrumentos; inexperiencia práctica; falta de tiempo y sobrecarga de trabajo. Los beneficios mencionados estaban relacionados con el formato claro y ordenado de los instrumentos; facilidad en la aplicación de pasos y seguridad en la práctica asistencial. Consideraciones finales: la implementación del Proceso de Enfermería en el sector ocurrió después de la identificación de los conocimientos previos del equipo de enfermería, seguido de la encuesta del perfil de los pacientes, creación de instrumentos, realización de educación permanente de los profesionales, aplicación y seguimiento en el uso de los instrumentos.

Descriptores: Proceso de Enfermería. Teoría de Enfermería. Registros de Enfermería. Terminología Normalizada de Enfermería. Educación Continua.

\section{Introdução}

A Enfermagem tem por finalidade assegurar o cuidado integral ao paciente de forma eficaz e organizada. A Sistematização da Assistência de Enfermagem (SAE) destaca-se neste sentido, orientando o planejamento, a execução e a documentação do cuidado prestado. Entre as vantagens da SAE está a possibilidade de dinamizar a prática profissional orientada pela gerência do Processo de Enfermagem (PE) ${ }^{(1)}$. Este é executado após a avaliação do paciente e possibilita colher informações que auxiliarão na identificação dos diagnósticos, que é a base para o planejamento das metas a serem alcançadas. As ações planejadas são implementadas por meio da execução dos cuidados prescritos. Na etapa seguinte, ocorre a avaliação das metas implementadas e se foram alcançadas ou devem ser retomadas as fases anteriores, no caso da identificação de novos diagnósticos, ou, ainda, se é possível avançar para o planejamento de novas metas ${ }^{(2)}$.

Em 2009, o Conselho Federal de Enfermagem (COFEN) revogou e atualizou a Resolução
COFEN $n^{\circ} 272$, de 2002, que instituiu a obrigatoriedade da implementação do PE em ambientes de saúde públicos e privados, onde ocorra a atuação de profissionais da enfermagem ${ }^{(3-4)}$.

Ainda que o PE seja respaldado por lei, existem fatores que dificultam a organização do trabalho por meio de um método, a exemplo de conhecimento teórico escasso ou ausente sobre o tema, inclusive durante a formação profissional, e insuficiência de tempo e de recursos humanos para a execução do cuidado nas instituições de saúde. Tais fatores, somados à falta de capacitação da equipe e à ausência de ferramentas adequadas para o registro da assistência, também contribuem para a não implantação do $\mathrm{PE}^{(5)}$.

Embora seja reconhecida a importância da SAE no processo do cuidar e em relação aos aspectos que sustentam a profissão, esse entendimento ainda não é consenso no meio profissional. Por esta razão, não está plenamente consolidado nos ambientes em que ocorre o cuidado de enfermagem ${ }^{(6)}$. 
É compreensível que, diante das dificuldades que permeiam a assistência de enfermagem, implementar um método assistencial não seja uma tarefa simples; porém é responsabilidade e dever dos profissionais de Enfermagem registrar, no prontuário, as informações inerentes ao processo do cuidar bem, como o gerenciamento dos processos de trabalho, para assegurar a qualidade da assistência ${ }^{(7)}$.

Reconhecendo a importância do cumprimento das recomendações legais e diante da realidade vivenciada nos serviços de saúde no tocante a esta temática, este estudo visou contribuir com o cenário estudado, pelo fato de tratar-se de hospital de ensino e campo de práticas e estágios para os cursos de Enfermagem. A descrição da operacionalização das etapas do PE torna possível compartilhar experiências e motivar sua realização em outros cenários. Desta forma, o presente estudo teve como objetivo descrever a implantação do Processo de Enfermagem em uma unidade de cuidados intermediários.

\section{Método}

Estudo do tipo pesquisa-ação, de abordagem qualitativa, realizado na Unidade de Cuidados Intermediários do Hospital Universitário da Universidade Federal do Vale do São Francisco (HU-Univasf). O serviço é de médio porte e atende 53 municípios que integram a Rede Interestadual de Atenção à Saúde do Vale do São Francisco (Rede PEBA), que compreende seis microrregiões de saúde com, aproximadamente, 2.068.000 habitantes dos estados de Pernambuco e Bahia. É referência no atendimento de urgência e emergência nos casos de: politraumatismos; neurologia; ortopedia; cirurgias geral, vascular e bucomaxilofacial; clínica médica e cirurgia plástica restauradora, com atuação multidisciplinar das equipes de saúde; além de procedimentos de alta complexidade, como neurocirurgias e cirurgias ortopédicas ${ }^{(8)}$.

O estudo ocorreu no período de dezembro de 2019 a março de 2020 e fez parte da pesquisa principal intitulada "Sistematização da Assistência de Enfermagem como Ferramenta para a Qualidade do Cuidado". A pesquisa-ação é um meio de desenvolvimento profissional, visto que há participação efetiva dos sujeitos envolvidos na prática, com vistas à mudança de comportamentos $^{(9)}$.

Desta forma, os pesquisadores conduzem o estudo, direcionando e mediando as atividades sugeridas, com o objetivo de buscar elementos de instrumentalização, com base nas discussões promovidas desde a primeira fase da pesquisa e nas teorias que as norteiam ${ }^{(10)}$. Desse modo, não somente geram dados para futuras intervenções ou recomendações ${ }^{(9)}$.

Este estudo relata a terceira e última fase de execução da pesquisa principal, cuja primeira fase avaliou o conhecimento dos profissionais de enfermagem da instituição selecionada sobre a $\mathrm{SAE}^{(11)}$. A segunda fase foi realizada por meio de um estudo qualitativo, que objetivou conhecer a percepção do profissional de enfermagem do serviço com relação à $\mathrm{SAE}$ e ao $\mathrm{PE}^{(12)}$. Resultou na elaboração dos instrumentos referentes à documentação do PE, todos baseados na Teoria das Necessidades Humanas Básicas de Wanda Aguiar Horta, implantados na última fase, objeto deste relato de experiência, a saber: Histórico de Enfermagem, Diagnóstico e Intervenção de Enfermagem e Avaliação de Enfermagem.

Para a operacionalização desta última fase, foram consideradas as peculiaridades referentes ao perfil da clientela atendida e à rotina instituída no setor. Assim, a Unidade de Cuidados Intermediários (UCI) foi selecionada para implantação dos instrumentos elaborados, por apresentar um quantitativo reduzido de pacientes, quando comparada a outros setores da instituição pesquisada. A amostra do estudo foi constituída por todos os profissionais de enfermagem atuantes no setor, sendo excluídos aqueles que se encontravam de férias ou afastados das suas atividades laborais no período da coleta.

Durante o processo de implantação, contou-se com o apoio do Núcleo de Educação Permanente em Saúde da instituição, que elaborou um plano educativo voltado à equipe de enfermagem da UCI. As atividades de capacitação foram realizadas em dezembro de 2019, de acordo com a disponibilidade dos profissionais. Ocorreram quatro encontros, com alcance 
de 17 profissionais, entre enfermeiros e técnicos de enfermagem atuantes no setor.

Os temas relativos ao $\mathrm{PE}$ e às Teorias de Enfermagem foram abordados nas aulas expositivas dialogadas, que tiveram duração média de uma hora. Os instrumentos confeccionados foram apresentados nesses momentos, assim como a sensibilização desses profissionais sobre a importância do seu uso. Após aplicação do plano educativo, os instrumentos foram implantados na UCI. O acompanhamento da implantação ocorreu nos meses de fevereiro e março de 2020, por meio de visitas previamente agendadas ao setor, com duração média de três horas de permanência, abrangendo todos os horários dos plantões e contemplando todos os enfermeiros. Durante o processo de implantação, foram realizadas seis visitas, nas quais era observado o preenchimento dos instrumentos em implantação pelos enfermeiros. À medida que as visitas ocorriam, os enfermeiros relatavam opiniões e críticas aos instrumentos ou à implantação do PE, sugestões e alterações que eram registradas por apenas um dos pesquisadores em diário de campo.

Durante as visitas in loco, os Técnicos de Enfermagem não apresentaram sugestões ou alterações. Eles participaram de todas as demais fases do estudo, como as reuniões de concepção, para implantação do PE na UCI, a capacitação e ainda no apoio ao preenchimento dos instrumentos. No entanto, os relatos de alterações para os pesquisadores foram feitos apenas por enfermeiros.

Nas referidas visitas, os dados relacionados à avaliação dos enfermeiros foram coletados por meio da técnica de observação participante, de forma sistematizada, com base em um roteiro contendo questões norteadoras que abordavam aspectos como: aplicabilidade/implantação dos instrumentos na rotina do setor, benefícios e dificuldades encontrados na sua utilização, sugestões para melhoria dos instrumentos e implicação da implantação do PE na qualidade da assistência. Nesses momentos, foi possível elucidar as dúvidas da equipe de enfermagem com relação aos instrumentos implantados no setor.

A coleta de dados foi interrompida quando ocorreu saturação teórica, isto é, quando, na visão dos pesquisadores, as informações repetiam-se e já não traziam elementos novos e relevantes ao objeto de estudo $^{(13)}$. Os dados foram analisados por meio da leitura dos conteúdos registrados no diário de campo, seguida da organização das informações numa sequência lógica ao planejamento e à execução das atividades estabele$\operatorname{cidas}^{(9)}$. Estas, em seguida, foram discutidas à luz da literatura científica a respeito do PE.

O estudo foi aprovado pelo Comitê de Ética em Pesquisa com Seres Humanos da Universidade de Pernambuco, CAAE n⿳o 57875216.2.0000.5207, obedecendo aos aspectos éticos da Resolução no 466, de 12 de dezembro de 2012, do Conselho Nacional de Saúde ${ }^{(14)}$. A participação da equipe de enfermagem ocorreu após a assinatura do Termo de Consentimento Livre e Esclarecido.

Visando garantir o anonimato dos participantes, eles foram identificados, neste texto, pela letra E (Enfermeiros) seguida de número arábico, correspondendo à identificação dos participantes.

\section{Resultados e Discussão}

Participaram do estudo 17 profissionais: 8 enfermeiros e 9 técnicos de enfermagem. Eram do sexo feminino 7 técnicos de enfermagem e 8 enfermeiros. Observou-se que, anteriormente à implantação, era utilizado um instrumento de Avaliação de Enfermagem parcial, pois não incluía as demais etapas do PE. Como consequência, havia falhas de registro, contrapondo-se ao preconizado pela legislação atual ${ }^{(7)}$. Os instrumentos para execução do PE foram criados com base na observação da escassez de informações presente na Avaliação de Enfermagem utilizada no setor, bem como em todo o hospital.

Estudo pondera que a utilização de instrumentos produzidos de acordo com a realidade da clientela atendida permite a observação mais detalhada e direcionada às necessidades individuais dos pacientes. Este modelo de assistência também contribui para o trabalho da gerência de enfermagem no momento da tomada de decisões, visto que são baseadas em informações mais completas e visíveis para toda a equipe ${ }^{(15)}$. 
Assim, nesta pesquisa, os novos instrumentos foram apresentados na UCI e contaram com a adesão dos profissionais da equipe de enfermagem quanto à sua utilização. No entanto, eles relataram alguns receios, sobretudo em relação à pouca experiência prática na utilização do $\mathrm{PE}$ e à sobrecarga de trabalho, uma vez que a rotina de banhos no leito e as evoluções eram divididas entre os horários diurno e noturno e as admissões concentravam-se no período noturno. Os relatos ilustram essa realidade.

Você vai me ajudar, pois só tive contato com isso durante a graduação. (E1).

A nossa equipe vai ficar sobrecarregada, porque as evoluções são feitas no momento do banbo e a gente tem mais banho para dar. (E2).

A maioria das admissões é feita durante nosso turno [noturno] [...] (E3).

É muita coisa para preencher. A gente vai ter que fazer todos os dias? (E4).

Em relação ao horário das Intervenções de Enfermagem, estudo afirma que podem ter a sua qualidade afetada, quando realizadas em um horário específico ou atribuídas exclusivamente a um turno do plantão, principalmente quando se tem um número reduzido de profissionais. Dessa forma, a falta de tempo e a sobrecarga de trabalho dos enfermeiros contribuem para a mecanização das intervenções e podem levar à repetição e consequente não compatibilidade com as necessidades do paciente ${ }^{(16)}$.

Apesar de todo processo de mudança gerar receios, dúvidas, preocupações e algumas resistências na aplicação de uma nova rotina, entende-se que a execução do PE precisa ser vista como facilitadora da atividade profissional. Estudo realizado em um hospital de São Paulo mostrou que a falta de conhecimento teórico e a sobrecarga de trabalho, devido à insuficiência de profissionais, são duas das principais barreiras para a aplicação do PE nos serviços de saúde ${ }^{(17)}$.

O plano educativo ofertado na instituição pesquisada teve a finalidade de capacitar as equipes que atuam na UCI para a utilização dos novos instrumentos. A realização das capacitações antes da implementação dos instrumentos no setor foi motivada pelo resultado de estudo realizado anteriormente no serviço, que evidenciou a falta de conhecimento dos entrevistados, no qual $59 \%$ dos profissionais de enfermagem souberam citar corretamente as etapas do PE, e 39,1\% souberam sequenciá-las corretamente ${ }^{(11)}$.

As capacitações, por meio de educação continuada sobre a temática abordada, têm como consequência a humanização e a qualificação na assistência de enfermagem. Esses tipos de qualificação auxiliam na atualização e no aprimoramento da formação dos profissionais, tornando-se fundamental na efetividade da assistência ${ }^{(18)}$.

À medida que, durante as visitas, aconteciam discussões sobre dificuldades encontradas quanto ao manuseio dos instrumentos, havia um sentimento de mudança e otimismo demonstrado pelos profissionais, que compreendiam a importância desse trabalho para a prática profissional e da união da equipe para o sucesso do processo de implantação no setor. Os relatos confirmam essa percepção:

Com o tempo, a gente pega. É questão de rotina. (E2).

No dia a dia, a gente vai acostumando-se. (E4).

O trabalho em equipe, na área da saúde, é imprescindivel e revela-se o caminho mais apropriado para o alcance de resultados positivos. A união dos profissionais com um mesmo propósito é traduzida em melhor desempenho individual, refletindo a qualidade da assistência prestada ao paciente que está sob seus cuidados ${ }^{(19)}$.

No decorrer do período de implantação dos instrumentos do PE, foi valiosa a participação dos profissionais de enfermagem em relação às sugestões e modificações propostas por eles. Embora tenham sido confeccionados de acordo com o perfil dos pacientes atendidos, os profissionais que atuavam no dia a dia no setor puderam contribuir para deixá-los completos e não haver inconsistências no momento da utilização.

$\mathrm{Na}$ primeira visita, após a implantação, um dos enfermeiros avaliou os instrumentos de forma positiva, mas sugeriu a alteração de alguns pontos. Entre eles, a retirada da opção "diurno" e "noturno" para os sinais vitais, visto que esses dados podiam ser colhidos em diferentes horários. Outra sugestão importante foi a inclusão da 
Escala de Sedação de Ramsay, devido ao perfil dos pacientes.

Estão bem completos, mas poderia melhorar em alguns pontos. A gente verifica os sinais vitais de acordo com a necessidade durante o plantão e não só uma vez. Os pacientes internados neste setor, em sua maioria, estão sob sedação. Então, seria importante ter a escala [de sedação]. (E1).

A UCI atende pacientes críticos, que se apresentam clinicamente descompensados, com patologias agudas ou crônicas, necessitando de monitoramento contínuo e suporte terapêutico avançado, tais como ventilação mecânica e drogas vasoativas ${ }^{(20)}$. No setor do estudo, os pacientes encontravam-se sedados, sendo necessária a utilização de meios artificiais de respiração. A aplicação de escala de sedação é amplamente recomendada em diretrizes de prática clínica, pois está associada a resultados positivos, no que diz respeito à avaliação frequente do nível de consciência ${ }^{(21)}$.

Ainda na primeira visita, outra observação realizada referiu-se às redundâncias de informações existentes nos instrumentos, tais como a verificação de edemas, tanto na Avaliação como nos Diagnósticos e Intervenções de Enfermagem, e ainda a retirada de diagnósticos que não se aplicavam ao perfil de pacientes atendidos naquele setor.

Na segunda visita, os instrumentos também foram avaliados positivamente. Observou-se alguns pontos a serem melhorados, como a organização das Intervenções de Enfermagem por ordem de prioridade, e redundâncias de informações já apontadas. A disposição das Intervenções de Enfermagem, de forma clara e ordenada, contribui para o planejamento integral da assistência. Esta organização antecipa a identificação das principais intervenções para determinada situação, facilita a sua definição e os recursos necessários para contemplá-las, tornado a assistência mais eficiente e produtiva ${ }^{(22)}$.

Ao longo da terceira visita, já com a inclusão das alterações sugeridas nas visitas anteriores, um dos enfermeiros aprovou os instrumentos, relatando que a nova forma de trabalho iria ajudar a focar mais no dia a dia. Apontou ainda a necessidade de formatação e organização, tais como a abreviação de alguns termos e a adição dos parênteses antes das opções a serem selecionadas. Houve a sugestão de aumentar o espaço para anotações de enfermagem e adicionar uma opção para caracterizar eventuais lesões por pressão:

As fichas têm tudo que a gente precisa. Vai melhorar a assistência, porque vai ficar mais completa. Melhor você organizar, para que não fique confuso na bora de preencher. A maioria dos pacientes, por ser paliativa, já chega ou acaba desenvolvendo LP [Lesão por Pressão]. (E3).

Para que o enfermeiro exerça seu trabalho com qualidade, é importante que ele tenha, além do aporte teórico, ferramentas adequadas para exercer a prática com segurança. A utilização de instrumentos é crucial para o registro do atendimento de enfermagem. Desse modo, a utilização de instrumentos inadequados dificulta a realização do PE, devido à falta de informações e à alta demanda de tempo do profissional responsável ${ }^{(5)}$.

$\mathrm{Na}$ quarta visita, os instrumentos já haviam sofrido as melhorias indicadas, porém foi incluída uma sugestão quanto à avaliação do cuff do traqueóstomo e do tubo orotraqueal, visto que a maioria dos pacientes fazia uso de ventilador mecânico. Além disso, foi sugerido que o aprazamento das Intervenções de Enfermagem fosse feito logo após os Diagnósticos de Enfermagem, em um espaço livre e sem divisórias de formatação.

É importante sempre observar o cuff, pra ver como ele está, e sempre anotar. (E1).

A manutenção da pressão correta do cuff está entre os cuidados de enfermagem com o paciente entubado, além de observar a fixação e trocar, quando necessário, realizar higiene oral e manter a cabeceira elevada. Garantir a manutenção da oxigenação de qualidade para o paciente, sobretudo naqueles em uso de tubo orotraqueal, é uma das atividades importantes do enfermeiro. Estes cuidados contribuem para a prevenção de complicações, como o desenvolvimento de lesões por pressão na mucosa traqueal e a ocorrência de microbroncoaspiração ${ }^{(23)}$.

No quinto momento de observação, dificuldades sobre a utilização dos instrumentos, principalmente no que se refere ao julgamento dos Diagnósticos de Enfermagem e ao aprazamento 
das Intervenções de Enfermagem, foram apontadas por um dos enfermeiros.

Eu não tenho muita afinidade com essa parte; você vai me ajudar. (E5).

Na prática assistencial, entre os fatores que representam dificuldades no sucesso da implementação do PE, estão a falta de conhecimento e preparo dos profissionais sobre seu manejo e a de material adequado para sua aplicação ${ }^{(6)}$. Um estudo que objetivou descrever a percepção e o nível de conhecimento de enfermeiros e acadêmicos de enfermagem brasileiros quanto ao PE, revelou que sua aplicação ou não aplicação está diretamente relacionada ao conhecimento sobre o tema ${ }^{(24)}$. Esse aspecto reflete a necessidade da busca por atualização, visto que a temática, quando abordada, tem maior ênfase durante a graduação $^{(25)}$.

Outra sugestão feita pelo mesmo enfermeiro foi a retirada do balanço hídrico do instrumento de Avaliação de Enfermagem, pois essa medida já era realizada pelos técnicos de enfermagem.

Os técnicos já fazem isso [balanço hídrico] na ficha deles. Quando a gente quer saber, a gente olha lá. Vai ficar muito repetitivo. (E5).

Além disso, também propôs a retirada da avaliação do volume de urina, apresentada no instrumento do Histórico de Enfermagem, já que não seria possível verificar com fidedignidade no momento da chegada do paciente, diante da possibilidade de alteração no setor de origem. Dessa forma, foram finalizadas as alterações dos instrumentos do PE.

A participação dos profissionais foi fundamental para o desenvolvimento deste estudo, tanto pela participação nas capacitações como na implantação dos instrumentos no setor, pelos enfermeiros. As suas contribuições auxiliaram a nortear a construção de instrumentos o mais próximo possível da realidade vivenciada por eles, para atender o perfil dos pacientes da unidade em questão. A maioria dos enfermeiros considerou que o PE seria essencial para a eficiência na assistência. Estudo afirma que a autonomia e a participação durante um processo de implantação podem contribuir para maior adesão dos profissionais na continuação da rotina de trabalho ${ }^{(24)}$.
Após o encerramento dos encontros, para readequação dos instrumentos e realização de todas as alterações sugeridas, foi realizada uma última visita, contemplando cada equipe do setor, para a verificação do andamento da implantação do PE. Os profissionais não declararam dificuldades no preenchimento e na realização das etapas do PE. Para confirmar a adesão da equipe aos instrumentos, dois meses depois do início da implantação no setor, foi observado o preenchimento de todos os instrumentos nos prontuários dos pacientes.

Uma limitação do estudo foi a dificuldade de implementar o plano educativo com os profissionais em cronograma viável para todos. A alta demanda de trabalho tornou necessária a readequação e replicação dos horários e dias estabelecidos no cronograma inicial.

Considera-se contribuição deste estudo ter permitido compreender e reafirmar que o estabelecimento de uma assistência sistematizada é um processo gradual que demanda, além de disposição pessoal, a união entre equipe assistencial e gestão diante do mesmo propósito, que é associar o cuidado qualificado ao paciente a um método de trabalho legalmente consolidado e seguro.

\section{Considerações finais}

A implantação do PE na UCI ocorreu após o conhecimento prévio da equipe de Enfermagem sobre a SAE, seguida do levantamento do perfil de pacientes da unidade, criação dos instrumentos do PE, realização de educação permanente dos profissionais, aplicação dos instrumentos e realização das alterações necessárias em conjunto com a equipe e, por fim, pelo acompanhamento da implantação.

A utilização de um método de trabalho organizado, no que diz respeito ao registro do cuidado de enfermagem, é parte fundamental entre as ações que contribuem para a garantia de uma assistência efetiva e de qualidade ao paciente. Foi possível perceber a adesão à utilização dos instrumentos implantados pela participação ativa da equipe no processo de mudança. Essa consequência reafirmou a necessidade do 
empoderamento da teoria sobre a temática, dispondo-se de ferramentas que viabilizem a prática assistencial qualificada.

Este estudo permitiu compreender e reafirmar que o estabelecimento de uma assistência sistematizada é um processo gradual que demanda, além de disposição pessoal, a união entre equipe assistencial e gestão diante do mesmo propósito, que é associar o cuidado qualificado ao paciente com base em um método de trabalho legalmente consolidado e seguro.

\section{Colaborações:}

1 - concepção, projeto, análise e interpretação dos dados: Flávia Emilia Cavalcante Valença Fernandes, Gerlene Grudka Lira e Rachel Mola;

2 - redação do artigo e revisão crítica relevante do conteúdo intelectual: Daislan Carvalho, Flávia Emília Cavalcante Valença Fernandes, Gerlene Grudka Lira e Rachel Mola;

3 - aprovação final da versão a ser publicada: Daislan Carvalho, Nêmora Lígia de Sousa Santana, Giselle Karine Muniz de Melo, Rita de Kacia de Sousa e Rachel Mola.

\section{Referências}

1. Ribeiro GC, Padoveze MC. Nursing Care Systematization in a basic health unit: perception of the nursing team. Rev Esc Enferm USP. 2018;52:e03375. DOI: http://dx.doi.org/10. 1590/S1980-220X2017028803375

2. Cruz DALM, Guedes ES, Santos MA, Sousa RMC, Turrini RNT, Maia MM, et al. Documentação do processo de enfermagem: justificativa e métodos de estudo analítico. Rev Bras Enferm. 2016;69(1):197-204. DOI: http:// dx.doi.org/10.1590/0034-7167.2016690126i

3. Conselho Federal de Enfermagem. Resolução COFEN $\mathrm{N}^{\circ}$ 358, de 15 de outubro de 2009. Dispõe sobre a Sistematização da Assistência em Enfermagem e a implementação do Processo de Enfermagem em ambientes públicos ou privados, em que ocorre o cuidado profissional de Enfermagem, e dá outras providências [Internet]. Brasília (DF); 2009 [cited 2020 Apr 15]. Available from: http://www.cofen.gov.br/resoluocofen-3582009_4384.html
4. Conselho Federal de Enfermagem. Resolução COFEN N 272, de 27 de agosto de 2002. Explicitou as etapas de implementação da Sistematização da Assistência de Enfermagem a ser aplicado pelo enfermeiro [Internet]. Brasília (DF); 2002 [cited 2021 Apr 30]. Available from: http://www.cofen.gov.br/resoluo-cofen-2722002revogada-pela-resoluao-cofen-n-3582009_ 4309.html

5. Silva FS, Carvalho Filha FSS. Sistematização da assistência de enfermagem a pacientes dialíticos: dificuldades, desafios e perspectivas. Arq ciênc saúde. 2017;24(2):33-7. DOI: http://dx.doi.org/10. 17696/2318-3691.24.2.2017.599

6. Garcia TR. Systematization of nursing care: substantive aspect of the professional practice. Editorial. Esc Anna Nery. 2016;20(1):5-10. DOI: http://dx.doi.org/10.5935/1414-8145.20160001

7. Conselho Federal de Enfermagem. Resolução COFEN N ${ }^{0}$ 429, de 22 de junho de 2012. Dispõe sobre o registro das ações profissionais no prontuário do paciente, e em outros documentos próprios da enfermagem, independente do meio de suporte - tradicional ou eletrônico [Internet]. Brasília (DF); 2012 [cited 2020 May 5]. Available from: http://www.cofen.gov.br/resoluocofen-n-4292012_9263.html

8. Brasil. Ministério da Educação. Empresa Brasileira de Serviços Hospitalares. Institucional. Nossa História [Internet]. Brasília (DF); 2020 [cited 2021 Apr 30]. Available from: https://www.gov. br/ebserh/pt-br/hospitais-universitarios/regiaonordeste/hu-univasf/acesso-a-informacao/ institucional

9. Engel GI. Pesquisa-ação. Educ rev. 2000;(16):181-91. DOI: http://dx.doi.org/10.1590/0104-4060.214

10. Cordeiro L, Soares CB. Processo de trabalho na Atenção Primária em Saúde: pesquisa-ação com Agentes Comunitários de Saúde. Ciênc Saúde Colet. 2015;20(11):3581-8. DOI: http://dx.doi. org/10.1590/1413-812320152011.10332014

11. Mola R, Dias ML, Costa JF, Fernandes FECV, Lira GG. The nursing professionals' knowledge with regards to the nursing care systematization. $\mathrm{J}$ res fundam care Online. 2019;11(4):887-93. DOI: http://dx.doi.org/10.9789/2175-5361.2019. v11i4.887-893

12. Nascimento ALG, Coelho EN, Fernandes FECV, Lira GG, Mola R. Percepção do profissional de enfermagem sobre a sistematização da assistência de enfermagem. Enferm Brasil. 2018;17(6):678-84. DOI: http://dx.doi.org/10.33233/eb.v17i6.2459 
13. Fontanella BJB, Ricas J, Turato ER. Amostragem por saturação em pesquisas qualitativas em saúde: contribuições teóricas. Cad Saúde Pública. 2008;24(1):17-27. DOI: http://dx.doi.org/10.1590/ S0102-311X2008000100003

14. Brasil. Conselho Nacional de Saúde. Resolução $\mathrm{N}^{\mathrm{O}}$ 466, de 12 de dezembro de 2012. Aprova as diretrizes e normas regulamentadoras de pesquisas envolvendo seres humanos [Internet]. Brasília (DF); 2012 [cited 2020 Apr 15]. Available from: https://conselho.saude.gov.br/resolucoes/2012/ Reso466.pdf

15. Benedet SA, Gelbcke FL, Amante LN, Padilha MIS, Pires DP. Nursing process: systematization of the nursing care instrument in the perception of nurses. J res fundam care online. 2016;8(3):4780-8. DOI: http://dx.doi. org/10.9789/2175-5361.2016.v8i3.4780-4788

16. Faeda MS, Perroca MG. Conformity of nurse prescribing to care needs: nurses' understanding. Rev Bras Enferm. 2017;70(2):400-6. DOI: http:// dx.doi.org/10.1590/0034-7167-2016-0107

17. Reis GS, Reppetto MA, Santos LSC, Devezas AMLO. Sistematização da assistência de enfermagem: vantagens e dificuldades na implantação. Arq Med Hosp Fac Cienc Med Santa Casa São Paulo [Internet]. 2016 [cited 2020 May 2]; 61:128-32. Available from: http://arquivosmedicos. fcmsantacasasp.edu.br/index.php/AMSCSP/ article/view/101

18. Moccelin JM, Pissaia LF, Costa AEK, Monteiro S, Rehfeldt MJH. A educação continuada como ferramenta de qualificação da equipe de enfermagem perante a avaliação da dor em idosos. Cad Pedagógico. 2017;14(2):161-76. DOI: http://dx.doi.org/10.22410/issn.1983-0882. v14i2a2017.1547

19. Laccort AA, Oliveira BG. A importância do trabalho em equipe no contexto da enfermagem. UNINGÁ Rev [Internet]. 2017 [cited 2020 May 7]; 29(3):6-10. Available from: http://revista.uninga.br/ index.php/uningareviews/article/view/1976/1572
20. Viana SSC, Arantes T, Ribeiro SCC. Interventions of the clinical pharmacist in an Intermediate Care Unit for elderly patients. Einstein (São Paulo). 2017;15(3):283-8. DOI: http://dx.doi.org/10.1590/ s1679-45082017ao3894

21. Namigar T, Serap K, Esra AT, Özgül O, Can ÖA, Aysel A, et al. Correlação entre a escala de sedação de Ramsay, escala de sedação-agitação de Richmond e escala de sedação-agitação de Riker durante sedação com midazolam-remifentanil. Braz J Anesthesiol. 2017;67(4):347-54. DOI: http:// dx.doi.org/10.1016/j.bjan.2017.03.006

22. Cruz ICF. Diagnósticos e Prescrições de Enfermagem: recriando os instrumentos de trabalho. Bol NEPAE-NESEN [Internet]. 2017 [cited 2020 Apr 15];14(1). Available from: http:// www.jsncare.uff.br/index.php/bnn/article/ view/3024/745

23. Santos C, Nascimento ERP, Hermida PMV, Silva TG, Galetto SGS, Silva NJC, et al. Boas práticas de enfermagem a pacientes em ventilação mecânica invasiva na emergência hospitalar. Esc Anna Nery. 2020;24(2):1-7. DOI: http://dx.doi.org/10.1590/2177-9465-ean-20190300

24. Oliveira MR, Almeida PC, Moreira TMM, Torres RAM. Nursing care systematization: perceptions and knowledge of the Brazilian nursing. Rev Bras Enferm. 2019;72(6):1547-53. DOI: http://dx.doi.org/10.1590/0034-7167-2018-0606

25. Silva VG, Cândido ASC. A formação do enfermeiro para a realização da educação continuada. Id on line Rev Mult Psic [Internet]. 2018 [cited 2020 Apr 15];12(40):847-58. Available from: https://idonline.emnuvens.com.br/id/article/ view/1162/0

Recebido: 12 de janeiro de 2021

Aprovado: 13 de maio de 2021

Publicado: 21 de dezembro de 2021

A Revista Baiana de Enfermagem utiliza a Licença Creative Commons - Atribuição-NãoComercial 4.0 Internacional.

https://creativecommons.org/licenses/by-nc/4.0/

Este artigo é de acesso aberto distribuído sob os termos da Licença Creative Commons (CC BY-NC).

Esta licença permite que outros remixem, adaptem e criem a partir do seu trabalho para fins não comerciais. Embora os novos trabalhos tenham de lhe atribuir o devido crédito e não possam ser usados para fins comerciais, os usuários não têm de licenciar esses trabalhos derivados sob os mesmos termos. 